\title{
TIC SEVERITY AND PSYCHOLOGICAL FUNCTION
}

The relationship between tic severity and neuropsychological function was examined in 12 monozygotic twin pairs with Tourette's syndrome at the National Institute of Mental Health, Bethesda, MD. Children with more severe tic symptoms had poorer overall performance on psychological tests, and differences were significant on individual tests of attention, visuospatial perception, and motor function. In each twin pair, the twin with more severe tics had poorer global neuropsychological function. (Randolph $\mathrm{C}$ et al. Tourette's syndrome in monozygotic twins. Relationship of tic severity to neuropsychological function. Arch Neurol July 1993; $\underline{50}$ : 725-728). (Reprints: Dr Randolph, Experimental Therapeutics Branch, National Institute of Neurological Disorders and Stroke, Bldg 10, Room 5C104, 9000 Rockville Pike, Bethesda, MD 20892).

COMMENT. Tic severity and psychological function in children with Tourette's syndrome appear to be influenced by nongenetic factors with a common pathophysiology. The authors found that disturbances of attention were a cardinal psychological feature of TS, but most neuropsychological measures were close to normal means.

\section{FEBRILE SEIZURES}

\section{DIAZEPAM PROPHYLAXIS OF FEBRILE SEIZURES}

The results of a double-blind, randomized, placebo-controlled trial of oral diazepam administered only at the time of fever are reported from Tufts University School of Medicine and Boston University School of Public Health, Boston. Among 406 children of mean age 24 months who received diazepam, $0.33 \mathrm{mg} / \mathrm{kg} /$ body $\mathrm{wt}$, or placebo orally every 8 hours during febrile illnesses, the reduction in risk of recurrence of febrile seizures in the diazepam-treated group, based on an intention-to-treat analysis, was $44 \%$ during a mean followup of 1.9 years. An analysis restricted to children who had seizures while actually taking the prescribed medication ( 45 seizures in 36 children) showed 29 seizure occurrences in the placebo group and only 7 in the diazepam group, an $82 \%$ reduction in recurrence risk. Moderate side effects, including ataxia, lethargy, or irritability, were reported in $59(39 \%)$ of 153 children who took at least one dose of diazepam. Mild side effects occurred with the same frequency. (Rosman NP et al. A controlled trial of diazepam administered during febrile illnesses to prevent recurrence of febrile seizures. $\mathrm{N}$ Engl I Med July 8 1993; 329: 79-84). (Reprints: Dr Rosman, Division of Pediatric Neurology, Floating Hospital for Children, New England Medical Center, 750 Washington St, Box 330, Boston, MA 02111).

COMMENT. The authors advocate the prevention of recurrence of all febrile seizures and recommend oral diazepam, taken at the first sign of 